\title{
O CONTROLE DA TRANSMISSÃO DA DOENÇA DE CHAGAS EM MAMBAÍ - GOIÁS, BRASIL (1982-1984)*
}

\author{
Marco Túlio Garcia-Zapata1, Philip Davis Marsden1, Domingos das Virgens1, Roberto \\ Penna1, Vânia Soares 1, Iberaci Americano do Brasil2, Cleudson Nery de Castro1, Aluizio \\ Prata ${ }^{1}$ e Vanize Macêdo'.
}

\begin{abstract}
A aplicaçâo de inseticidas em Mambaí-GO, desde 1980, está determinando uma diminuição progressiva inicial de Triatoma infestans no intradomicílio, mas não a sua eliminação. A infestação triatomínica foi detectada atra vés de diversos métodos de vigilância imediata (transversal) e a longo prazo (longitudinal), com a colaboração dos próprios moradores. No primeiro ano de controle foi observada uma queda significante de $28,6 \%$ a 13,5\%, mas devido a uma falha no programa de expurgos, em 1981, esta cifra voltou a elevar-se $(23,2 \%)$. A continuidade desses expurgos nos anos seguintes resultou em um decínio gradual, atingindo em 1984 o nivel de $14,2 \%$. Simultaneamente a percentagem intradomiciliar de T. sordida tendeu a aumentar, embora a infecção tripanossômica tenha sido sempre mínima. O conjunto destes achados sugerem que o controle do $\mathrm{T}$. infestans com o uso exclusivo de inseticidas ( $B H C$ e Deltametrina) é dificil e oneroso. Precisando-se, portanto, o uso de medidas supletivas integradas aos sistemas de controle de doença de Chagas, que encorajam a participação ativa das comunidades afligidas, estimuladas mediante programas educativos.
\end{abstract}

Palavras chaves: Trypanosoma cruzi. Triatoma infestans. Triatoma sordida. Inseticidas. Controle da doença de Chagas.

Foram publicados, anteriormente, diversos trabathos, que descrevem a situação ecológica do Triatoma infestans no municipio de Mambai-GO, onde foi observado um aumento significante da infestação triatominica domiciliar (1975-1979) 911 . Assim sendo e por solicitação do projeto, em 1980, a SUCAM realizou o ataque maciço com insetiridas, sendo que uma metade dos domicílios recebeu a repetição de uma borrifação ${ }^{8}$. De forma seguida, foram também, relatados os resultados obtidos nos dois primeiros anos desta atividade ${ }^{12}$.

O propósito deste trabalho é dar continuidade ao estudo do programa de controle da transmissão da doença de Chagas, no municipio acima referido, dando ênfase ao período de 1982-1984.

\section{MATERIAL E MÉTODOS}

As fases preparatórias e de ataque, foram executadas segundo a regulamentação e normas técnicas da SUCAM - Superintendência de Campanhas de Saúde Pública - Divisão de Doença de Chagas, Ministério da Saúde do Brasil 122021.

\footnotetext{
1. Núcleo de Medicina Tropical e Nutrição, Universidade de Brasilia.

2. Superintendência de Campanhas de Saúde Pública (SUCAM), Ministério da Saúde.

* Trabalho financiado pelo Conselho Nacional de Desenvolvimento Científico e Tecnológico ( $\mathrm{CNPq} / \mathrm{PIDE}$ ); Ministério da Saúde (SUC AM) e UNDP/World Bank/WHO-TDR. Recebido para publicação em 9/5/1986
}

Na fase inicial, a área foi mapeada determinando-se 58 localidades denominadas "fazendas", que ficaram reduzidas a 56 desde 1982. Para este estudo foi apenas considerado o acompanhamento evolutivo anual das 724 unidades domiciliares rurais pesquisadas em 19805812. Cada uma dessas foi examinada para determinar a infestação triatomínica, mediante um sistema de vigilância implantado em 1981, e realizado por duas equipes: SUCAM e UnB (Universidade de Brasilia), ambas realizaram, em separado, uma captura manual com insetifugos (mistura 1: 8 de piretro e butóxido de piperonil). Além disso, a equipe da UnB, acompanhou todas as casas que na fase inicial apresentaram evidència de infestação triatominica, mediante a utilização de "unidades de vigilância" 10 , associação de uma caixa de GómezNuñez, sacola de plástico e cartaz informativo. Cada uma destas unidades foi examinada e trocada pelo menos uma vez por ano. $A$ avaliação destas técnicas de vigilância será trátado em separado.

Foram consideradas infestadas, apenas aquelas unidades domiciliares com captura efetiva de triatomineos (seja pelo guarda da SUCAM e/ou UnB ou pelos moradores) e/ou presença de vestígios (cascas, ovos ou fezes de triatomineos) no interior das caixas de Gómez-Nuñez. Em cada uma destas unidades, com infestação residual, a SUCAM aplicou um expurgo seletivo, tanto no intra quanto no peri-domicilio (galinheiros).

Até o primeiro semestre de 1983, o inseticida usado foi o BHC (Organoclorado, OMS-0017) com 
Garcia-Zapata MT, Marsden PD, Virgens D, Penna R, Soares V, Brasil IA, Castro CN, Prata A, Macêdo V. O controle da transmissão da doença de Chagas em Mambai-Goiás, Brasil (1982-1984). Revista da Sociedade Brasileira de Medicina Tropical 19: 219-225, Out-Dez, 1986.

$30 \%$ de isómero gamma, na dosagem de $0,5 \mathrm{~g} / \mathrm{m}^{2}$. Posteriormente e até o momento se vem utilizando Deltametrina (inseticida piretróide, OMS-1998) na dosagem de $0,05 \mathrm{~g} / \mathrm{m}^{2}$. Entretanto, no inicio de 1984 , 22 casas foram borrifadas com Malathion (organofosforado, OMS-0001) na dosagem de $2,5 \mathrm{~g} / \mathrm{m}^{2}$, por falta de recursos, na época, para compra de Deltametrina.

Por outro lado, todos os triatomineos capturados através das técnicas acima referidas, foram identificados e examinados em laboratório, para determinar-se a taxa de infecção tripanossômica, por espécie e estágio.

Igualmente, em 1984, foi implantado um programa educativo e de participação comunitária em 12 localidades do município, objetivando outras publicações 67 .

Para fins avaliativos do programa de controle do municipio todo, empregaram-se os dados obtidos anualmente pelas equipes da UnB e SUCAM, em conjunto e isoladamente, e calcularam-se alguns dos indicadores entomológicos sugeridos pela Organização Mundial da Saúde 22.

Da mesma maneira, acompanhou-se anualmente 686 casas borrifadas, sendo que 465 receberam uma, e 221 duas borrifações respectivamente, das 724 selecionadas inicialmente para o estudo longitudinal. Nestas, considerando que desde 1981, a norma de borrifação era seletiva, analizaram-se vários critérios para o estudo do efeito da repetição de uma borrifação no início de um programa de controle da doença de Chagas: \% de infestação geral e especifica para $T$. infestans, indice de borrifação geral e seletivo para $T$. infestans e indice de cobertura. Além disso, avaliou-se a freqüência de infestação intradomiciliar para $T$. infestans em um grupo selecionado de 150 domicilios que apresentaram infestação consecutivamente nos dois anos que antecederam a fase de ataque maciço. Avaliou-se retrospectivamente 40 unidades domiciliares infestadas com $T$. infestans em 1984, que foram devidamente acompanhadas através de todos os métodos de vigilância, implantados na área desde $1981^{512}$.

No estudo da dinâmica de infestação anual dos domicilios utilizaram-se os dados obtidos pelas duas equipes em conjunto (UnB/SUCAM), sendo pesquisados no total 2142 intradomicílios no periodo 1980 1984. Os critérios de invasão, reinfestação e persistência aqui empregados, correspondem aos referidos por Soler e cols ${ }^{19}$ e Garcia-Zapata .

Para a determinação da eficiência do programa de vigilância, empregaram-se diversos critérios: a) avaliação longitudinal do programa geral ( $\mathrm{UnB}$ / SUC AM); estudo de 271 intradomicilios desde a fase pré-borrifação ate 1984 que foram acompanhados através de todos os métodos de vigilância;b) avaliação da detecção de intradomicilios infestados por $T$. infestans segundo equipe de trabal ho no periodo 1981 1984 ; c) avaliação da dinâmica da população triatomínica e a sua relação com a transmissão da doença de Chagas no decurso do programa de controle.

Finalmente, determinaram-se os custos gerados no programa de vigilância epidemiológica no periodo de 1980-1984, a partir de informes proporcionados pela SUCAM e do banco de dados do Projeto Mambai da UnB.

A análise estatística dos dados foi realizada pelo Centro de Processamento de Dados (CPD) da Universidade de Brasilia. Foram calculados os seguintes testes não paramétricos: teste do qui-quadrado $\left(\mathrm{X}^{2}\right)$ para duas amostras independentes e teste entre duas proporçōes. $O$ nivel de significância dos testes $(\alpha)$, geralmente, foi de $5 \%$. No entanto, em casos específicos e a critério dos autores, assinalou-se um nivel diferente, com a finalidade de permitir uma melhor identificação do fenômeno observado.

\section{RESULTADOS}

Depois da fase de ataque maciço em 1980, as unidades domiciliares infestadas, em geral e localizadas na área rural, sofreram uma queda significante no nivel de infestação em $1981\left(\mathrm{P}_{1}>\mathrm{P}_{2}, \alpha 0,05\right)$, no entanto em 1982 esta cifra elevou-se a niveis muito próximos aos observados em $1980\left(\mathrm{P}_{1} \cong \mathrm{P}_{2}, \alpha 0,05\right)$. Nos últimos dois anos a percentagem da infestação diminuiu leve e gradativamente, sendo que o nivel de infestação de 1984, foi significativamente menor que o de $1980\left(\mathrm{P}_{1}>\mathrm{P}_{2}, \alpha 0,05\right)$.

Quando analisaram-se os intradomicilios infestados por $T$. infestans, observou-se também uma situação parecida com a descrita para a infestação em geral. No relacionado com a infestação intradomiciliar por $T$. sordida, esta permaneceu oscilante em niveis inferiores aos observados para $T$. infestans mas com tendência a aumento progressivo. Nos peri-domicílios, $T$. infestans sempre permaneceu em niveis baixos e não significantes, $T$. sordida apresentou caracteristicas semelhantes a infestação geral (Tabela 1).

Ao considerar-se a borrifação anual dos intradomicilios infestados com $T$. infestans, observou-se um decréscimo significante na cobertura $(53,2 \%)$ para o ano de $1981\left(\mathbf{P}_{1}>\mathbf{P}_{2}, \alpha 0,05\right)$. No período de 19821984, a percentagem de intradomicilios borrifados sempre foi superior a $90 \%$, no entanto nunca atingiu o nivel de cobertura da fase de ataque maciço em 1980 $(98,6 \%)$.

$\mathrm{Na}$ avaliação dos indicadores entomológicos obtidos, tanto no grupo de estudo longitudinal na área rural (UnB-SUCAM) quanto no municipio todo (SUCAM), observou-se os mais diversos padrões de comportamento. Nestes destacou-se: a) tendência a 
Garcia-Zapata MT, Marsden PD, Virgens D, Penna R, Soares V, Brasil IA, Castro CN, Prata A, Macêdo V. O controle da transmissão da doença de Chagas em Mambai-Goiás. Brasil (1982-1984). Revista da Sociedade Brasileira de Medicina Tropical 19: 219-225, Out-Dez. 1986.

Tabela 1 - Prevalência de infestação intra e peridomiciliar detectada pelas duas equipes UnB e SUCAM de forma conjunta na área rural de Mambaí - GO (1980-1984).

\begin{tabular}{lccccc}
\hline \multirow{2}{*}{\begin{tabular}{l} 
Estudo de \\
\cline { 2 - 6 }
\end{tabular}} & Unidades Domiciliares & \multicolumn{2}{c}{ Intradomicilios infestados } & \multicolumn{2}{c}{ Peridomicilios infestados } \\
\cline { 2 - 6 } & Pesquisadas (\%) & T. infestans (\%) & T. sordida (\%) & T.infestans(\%) & T. sordida(\%) \\
\hline 1980 & $724(100,0)$ & $207(28,6)^{\mathrm{a}}$ & $32(4,4)$ & $9(1,2)^{\mathrm{e}}$ & $57(7,9)^{\mathrm{i}}$ \\
\hline 1981 & $695(100,0)$ & $94(13,5)^{\mathrm{b}}$ & $23(3,3)$ & $4(0,6)^{\mathrm{f}}$ & $47(6,8)$ \\
\hline 1982 & $563(100,0)$ & $131(23,2)^{\mathrm{c}}$ & $31(5,5)$ & $3(0,5)^{\mathrm{g}}$ & $56(9,9)$ \\
\hline 1983 & $580(100,0)$ & $99(17,1)$ & $28(4,8)$ & $19(3,3)$ & $62(10,7)$ \\
\hline 1984 & $570(100,0)$ & $81(14,2)^{\mathrm{d}}$ & $32(5,6)$ & $8(1,4)^{\mathrm{h}}$ & $62(10,9)^{\mathrm{j}}$ \\
\hline
\end{tabular}

Análise estatística das proporçoes: $\mathrm{P}_{1} \neq \mathrm{P}_{2}(\alpha 0,05):$ a \& b, a \& d, i \& j, a \& c

$\mathrm{P}_{1} \cong \mathrm{P}_{2}(\alpha 0,05): \mathrm{b} \& \mathrm{~d}$, e \& $\mathbf{f}$, e $\& \mathrm{~h}$, e \& $\mathrm{g}$

diminuir dos seguintes indicadores: \% de infestação triatomínica, indice de densidade triatomínica intradomiciliar, índice de aglomeração intradomiciliar e \% de infestação intradomiciliar de $T$. infestans; e b) a tendência a aumentar dos seguintes indicadores: indice de dispersão de triatomineos no geral, \% de infestação intradomiciliar de $T$. sordida e índice de dispersão de $T$. sordida.

$\mathrm{Na}$ avaliação dos diferentes indices de infestação, borrifação e cobertura no decurso dos anos de 1980 a 1984, no grupo I, com só uma borrifação e no grupo II, com duas borrifações, observou-se os seguintes resultados: a) a \% de infestação domiciliar em geral, na fase de borrifação seletiva foi sempre predominante no grupo I $\left.\left(\mathrm{P}_{1}>\mathrm{P}_{2}, \alpha 0,05\right) ; \mathrm{b}\right)$ nos outros indices, que refletem o grau de infestação domiciliar por $T$. infestans e as borrifações por ela geradas, sempre mostraram um maior predominio no grupo II $\left(\mathrm{P}_{1}>\mathrm{P}_{2}, \alpha 0,05\right)$, com á única exceção do indice de borrifação domiciliar do ano de $1983 ;$ c) o índice de cobertura das borrifações anuais, foi bastante irregular, no entanto, com a exceção de 1981 , sempre foi acima de $70 \%$. Da mesma maneira, na análise longitudinal dos grupos selecionados de casas infestadas por $T$. infestans, mostraram paradoxalmente maiores benefícios no grupo I. Revelando, em sintese, a não superioridade do grupo II, de duas borrifações, em relação ao grupo I, de uma borrifação.

A constatação, de invasão anual dos intradomicilios por T. infestans, teve um curso oscilante, que em média foi de $10,6 \%$. Por outro lado, a percentagem dos intradomicílios que persistiu com $T$. infestans teve uma média anual de 5,7\%. Nu relacionado com $T$. sordida a média de invasão e persistência anual foram de $3,4 \%$ e de $1,1 \%$ respectivamente. A substituição de uma espécie por outra foi constantemente baixa. Mais detalhes na Tabela 2.

Tabela 2 - Estudo longitudinal, anual dos intradomicilios na área rural de Mambai - GO (1980-1984) UnB/SUCAM.

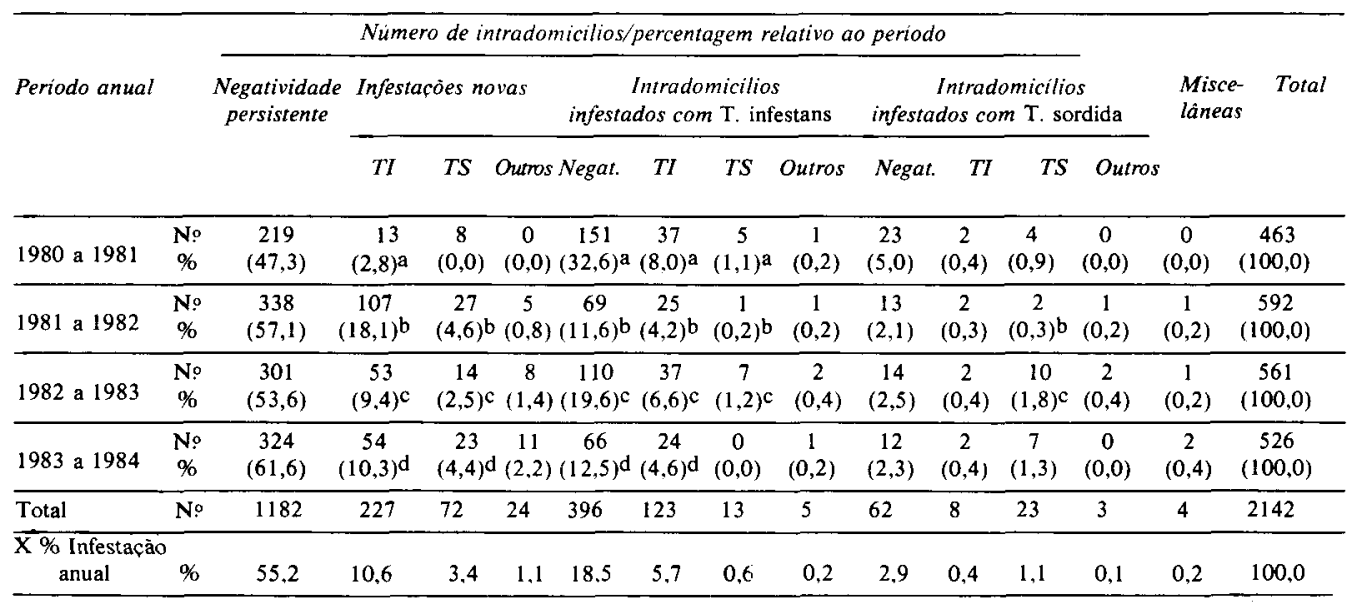

$\mathrm{TI}=T$. infestans $\quad \mathrm{TS}=T$. sordida

Análise estatística das proporçōes $\mathrm{P}_{1} \neq \mathrm{P}_{2}(\alpha 0.05)$ : a \& b, b \& c, a \& d. 
Garcia-Zapata MT, Marsden PD, Virgens D, Penna R, Soares V, Brasil IA, Castro CN, Prata A, Macêdo V. O controle da transmissão da doença de Chagas em Mambai-Goiás, Brasil (1982-1984). Revista da Sociedade Brasileira de Medicina Tropical 19: 219-225, Out-Dez, 1986.

$\mathrm{Na}$ avaliação a longo prazo de 271 intradomicilios, constatou-se que: a) a carga de infestação triatomínica dos intradomicilios infestados com $T$. infestans diminuiu gradativamente no decurso dos anos. No ano de 1980 , foram detectados $25,6 \%$ (31/121) dos intradomicilios com mais de dez triatomineos, enquanto $\mathrm{em} 1984$ foram apenas $5,0 \%(2 / 40)$; b) a maior percentagem de infestação de intradomicilios em 1984, derivou-se do grupo que na fase pré-ataque tinha mais de 25 triatomineos por intradomicilio, 36,8\% (7/19). No entanto, no exame acurado deste mesmo grupo, observou-se que nos 40 intradomicílios infestados com T. infestans em 1984: $16(40,0 \%)$ corresponderam à invasão, $13(32,5 \%)$ à reinfestação, $10(25,0 \%)$ à persistência, e apenas um em que se teve dúvida porque estando infestado com $T$. sordida, alternou um periodo negativo antes da infestação por $T$. infestans (Fig. 1).

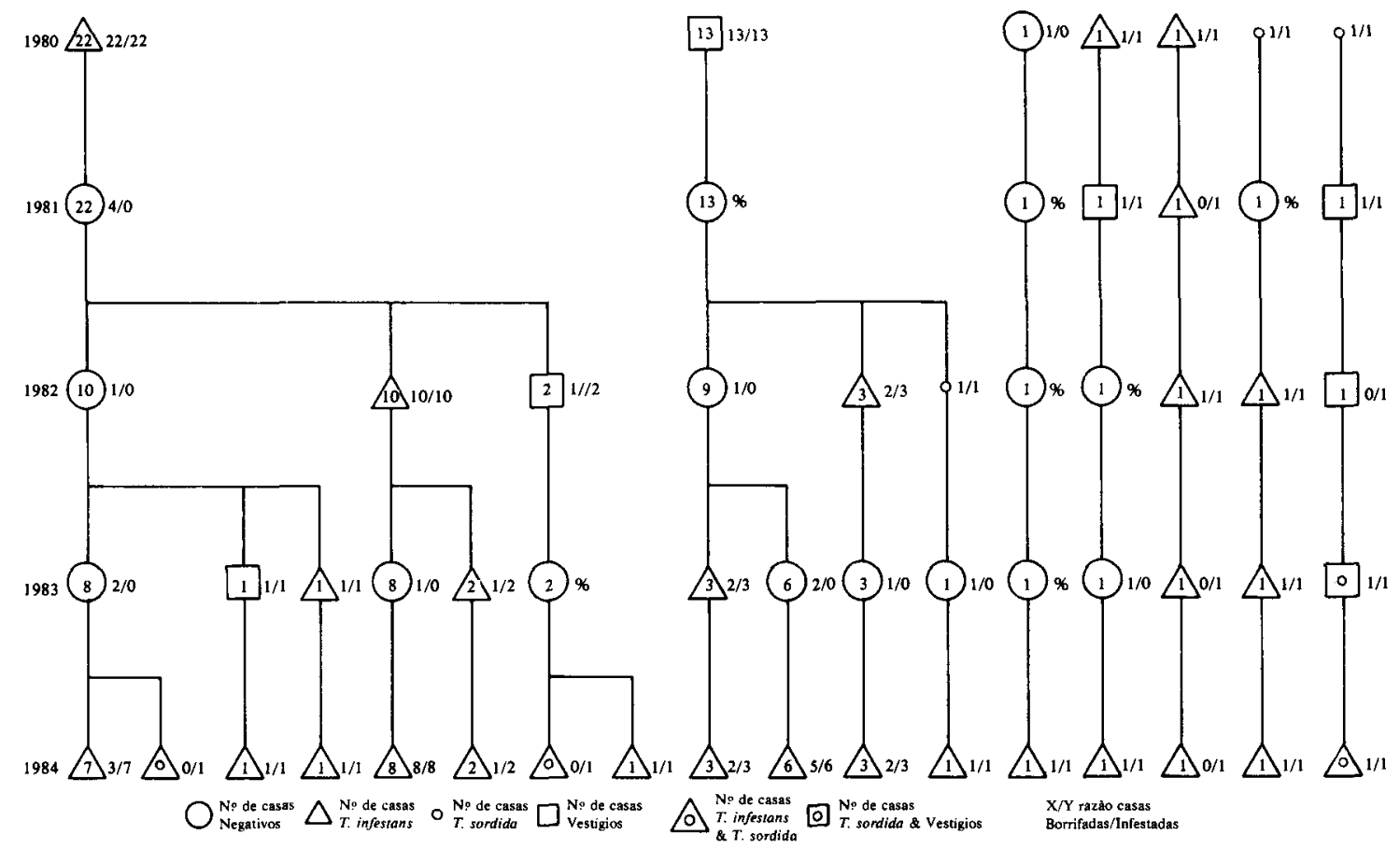

Fig. 1 - Diagrama do histórico retrospectivo de 40 casas infestadas com T. infestans em 1984. Mambai - GO (1980-1984).

No periodo de 1981-1984, a equipe da UnB isoladamente conseguiu detectar através dos seus métodos de vigilância (captura manual, caixa GómezNuñez e notificações), o maior número de intradomicilios infestados por $T$. infestans com uma média anual de $17,3 \%(342 / 1976)$ enquanto só $4,7 \%$ (104/ 2220) foram detectados pela equipe da SUCAM mediante apenas uma captura manual.

Por outro lado, na avaliação da dinâmica da população triatominica e a sua relação com a transmissão da doença de Chagas, destacaram-se: a) diminuição do indice de captura manual de triatomineos vivos de 14,3 (1417/99) na fase pré-borrifação, para $1,6(65 / 40)$ no ano de $1984 ;$ b) diminuição do índice de notificações de $T$. infestans de $1,35(187 / 139)$ no ano de 1981, e 1,2 (41/34) no ano de 1984.

No que diz respeito à infecçào tripanossômica $\mathrm{em}$ triatomineos, no grupo de estudo longitudinal (UnB-SUCAM) foram apenas realizadas duas avalia- ções: $6,3 \%(11 / 175)$ no ano de 1981 e 4,9\%(11/226) no ano de 1984 , detectados unicamente em espécimes de $T$. infestans. Também neste mesmo ano apenas um exemplar de $T$. costalimai (macho) capturado no intradomicílio encontrou-se infectado com formas semelhantes ao Trypanosoma conorhini. Os 69 exemplares de $T$. sordida examinados em 1984 foram todos negativos. Entretanto, a SUCAM no total do municipio observou o seguinte: $4,3 \%(11 / 256)$ no ano de $1981 ; 0,4 \%(1 / 224)$ em $1982 ; 0,0 \%(0 / 64)$ em 1983 e $0,9 \%(2 / 218)$ em 1984 . Aqui os espécimes examinados e positivos foram oito $T$. sordida e três $T$. infestans no ano de 1981, uma ninfa no ano de 1982 e um exemplar de $T$. sordida e de $T$. infestans no ano de 1984.

$\mathrm{Na}$ determinação dos custos do programa, constatou-se que: a) a aplicação de inseticidas no periodo de 1982-1984, demandou um custo médio real por unidade domiciliar trabalhada (em \$USA) oscilante 
Garcia-Zapata MT, Marsden PD, Virgens D, Penna R, Soares V, Brasil IA, Castro CN, Prata A, Macèdo V. O controle da transmissào da doença de Chagas em Mambai-Goiás, Brasil (1982-1984). Revista da Sociedade Brasileira de Medicina Tropical 19: 219-225, Out-Dez, 1986.

entre 5,5 e 19,8; sendo que o maior ônus percentual foi representado pelas diárias, $75,6 \%$, para o ano de 1982 e os inseticidas, $67,4 \%$ e $53,5 \%$, para os anos de 1983 e 1984, respectivamente (Fig. 2); b) o custo médio da captura manual com insetifugos por unidade domiciliar foi (\$USA): 9,4 (1982); 1,2 (1983) e 15,0 (1984); c) o custo da unidade de vigilància foi (\$ USA): 1,0 (1984).

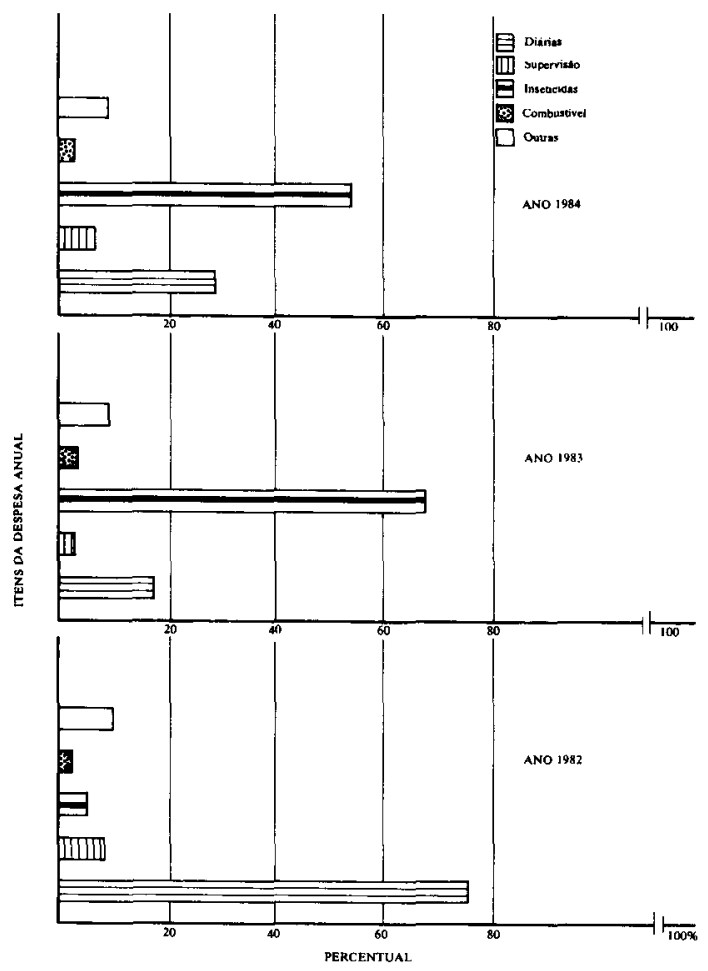

Fig. 2 - Custos do programa de controle da doença de Chagas: Combate com inseticidas. Percentual da despesa anual. Mambaí - GO - SUCAM (19821984).

\section{DISCUSSĀO}

Embora, tenham sido relatados, previamente, os resultados obtidos no primeiro ano de controle da doença de Chagas em Mambai - GO ${ }^{8}{ }^{12}$, considera-se importante um acompanhamento mais prolongado da evolução da dinâmica de um programa de vigilância epidemiológica, pois são informações muito valiosas, no entanto escassas na literatura existente.

$\mathrm{Na}$ avaliação final desses resultados, deve-se considerar que os diferentes inseticidas utilizados nos expurgos, pela SUCAM (BHC, Deltametrina e Malathion) durante o desenvolvimento deste estudo, constituíram uma variável incontrolável. Esses fatos devem refletir as dificuldades que enfrentariam programas semelhantes a nivel nacional diante da falta de recursos, de maneira semelhante, como aconteceria em outros países endêmicos, e, menos desenvolvi$\operatorname{dos}^{13}$.

Um aspecto interessante a destacar neste trabalho, é que o Projeto Mambai representa, na atualidade, um estudo continuo e contíguo de cada uma das casas que foram avaliadas na fase inicial do estudo e que sâo acompanhadas ano a ano pela nossa equipe técnica. $O$ regime aplicado na fase de ataque foi de apenas um expurgo maciço na totalidade das localidades infestadas, seguido de borrifaçōes adicionais seletivos. Da mesma maneira, dispomos de registro anual e por domicilio, referente a quantidade de inseticida gasto na campanha. Todos esses fatos o diferenciam de outros estudos desta natureza 2417 .

Da mesma forma, deve-se salientar, que o principio básico deste estudo, foi de avaliar o comportamento do $T$. infestans diante da aplicação de medidas de controle, por tratar-se, este, de um adequado modelo de espécie triatomínica, pela sua ampla distribuição na América Latina, pela sua estrita domiciliaridade e pela grande susceptibilidade de infecção pelo $T$. cruzi $i^{13}$. Podendo entretanto, também ser aplicadas em áreas endêmicas com espécies triatominicas de comportamento semelhante, como seria o caso de $P$. megistus intradomiciliar no nordeste do Brasil.

$O$ fato que depois da fase de ataque maciço tenha ocorrido uma queda significativa na prevalência da infestação das unidades domiciliares em 1981 , seguido de uma recuperação notável da população triatomínica no ano de 1982, coincidente, com uma baixa na cobertura de borrifações no ano prévio, demonstraram categoricamente, que a quebra na continuidade de um programa de controle pode determinar a reinfestação de casas expurgadas. Mostrando a necessidade de se manter a sequeencia desses programas, de forma continua, para a obtenção do sucesso esperado. Reduzindo ao minimo o nivel de transmissão da doença de Chagas.

A prevalència atual de $T$. infestans nesta área mostrou que o uso exclusivo de inseticidas da maneira como é empregado, não é suficiente. Pois não obstante, se consiga reduzir os indices de infestação triatomínica domiciliar, com muita dificuldade se consegue atingir o nivel sugerido como ideal para interromper a transmissão da doença de Chagas. Também, aqui, deve-se considerar o fato de que essa prevalència seja resultado do emprego de múltiplos métodos de vigilância, altamente sensiveis, que revelaram inclusive baixos nive is de infestação, não detectados pelo método rotineiro da captura manual, contrário ao referido por Piesman e Sherlock ${ }^{14} \mathrm{em}$ área de $P$. megistus. Contudo, os indicadores entomológicos, em nossa área de estudos, mostraram uma tendência a diminuição progressiva do $T$. infestans, porém, o indice de dispersão e a percentagem de infestação intradomiciliar de $T$. sordida tenderam a aumentar. 
Garcia-Zapata MT, Marsden PD, Virgens D, Penna R, Soares V, Brasil IA, Castro CN, Prata A, Macêdo V. O controle da transmissāo da doença de Chagas em Mambai-Goiás, Brasil (1982-1984). Revista da Sociedade Brasileira de Medicina Tropical 19: 219-225. Out-Dez, 1986.

O aumento de $T$. sordida, embora não alarmante pela reduzida taxa de infestação tripanossômica, talvez, possa ser explicado pelo fato de que a diminuição da população predominante e bem adaptada ( $T$. infestans) deu oportunidade para que esta espécie, competidora, começasse a se estabelecer. Este fato, já foi observado nas fases iniciais do estudo ${ }^{12}$. Da mesma maneira, como outros pesquisadores, relataram semelhante ocorrência em áreas onde fora erradicado o $T$. infestans.

Por tudo o referido, mediante a aplicação deste programa de vigilância, tem-se evidências claras de que o nivel de transmissão vetorial da doença de Chagas em Mambai - GO está caindo, fato demonstrado pela queda da carga triatominica e de infecção tripanossômica do $T$. infestans. No entanto, para declarar que definitivamente atingiu-se o nivel ideal no controle deste vetor, se realizará, em breve, um inquérito sorológico em todas as crianças nascidas depois da fase de ataque com inseticida.

Por outro lado, na avaliação do efeito da repetiçăo de uma borrifação no inicio de um programa de controle da doença de Chagas na permanência da infestação residual, pode-se inferir que o efeito das duas borrifações seja, apenas, válido para o periodo imediato à fase de ataque maciço. Porém, Marsden e $\mathrm{cols}^{12}$, no primeiro ano deste estudo, também não relataram beneficios no emprego desta metodologia. Sugerindo, que é, ainda, discutivel a validade das duas borrifações, no início do programa de controle, proposto pioneiramente por Dias ${ }^{1}$. Entretanto, tratandose de um assunto de muita polêmica, este procedimento deve ser reavaliado, com o objetivo de determinar sua evidência benéfica, caso contrário, deve ser evitado, pois os elevados custos destas campanhas geralmente acarretam a descontinuidade e fracasso das mesmas.

No estudo da dinâmica da infestação anual dos domicílios, conseguiu-se concluir que, ao contrário do primeiro ano de vigilância, a persistência de $T$. infestans intradomiciliar esteve mais ligada à invasão de domicílios que à persistência de populações residuais 512 . Concordando com as afirmaçōes de Schofield e Natthews ${ }^{16}$, a respeito da possibilidade de invasão triatominica procedente de casas que escaparam ao tratamento com inseticidas localizadas dentro do perímetro dos 200 metros.

$\mathrm{Na}$ avaliação dos custos, semelhante ao relatado por outros autores ${ }^{18}$, observou-se que dois são os fatores que determinam a elevação dos ônus nos programas de controle nacionais: a) manutenção das equipes de trabalho na área de campo; b) tipo de inseticida empregado (Deltametrina e Malathion, são mais caros que o BHC). Entretanto, não se encontrou uma explicação clara para as variações anuais de custos, tendo em vista que não necessariamente acompanharam a evolução inflacionária governamental.
Dessa maneira, com a finalidade de obter uma metodologia adequada e eficaz no controle desta doença, acha-se necessário um enfoque amplo e integrado das medidas de controle. $\mathrm{O}$ uso de inseticidas deve ser racional, contínuo, contiguo e apoiado por técnicas de vigilância preferencialmente a longo prazo (longitudinais), como as "unidades de vigilância"10, pela sua alta sensibilidade, que detectarão triatomineos com maior freqüência e a mais baixo custo que a tradicional captura manual. De forma concomitante devem ser implementadas medidas supletivas que estimulem a participação ativa das próprias comunidades, mediante programas educativos sanitários ${ }^{6} 7$.

\section{$S U M M A R Y$}

The spraying with insecticides in Mambai $G O$ in 1980 caused a progressive decrease of intradomiciliary $\mathrm{T}$ infestans but not its eradication. With the collaboration of the local population triatomine infestation was detected using several vigilance methods. In the first year of control there was a significant decrease from $28.6 \%$ to $13.5 \%$, but because offailures in the spraying program during 1981, this level increased to $23.2 \%$. The continuation of selective spraying in subsequent years resulted in a progressive fall, and in $198414.2 \%$ of the houses registered bugs. Simultaneously the percentage of intradomiciliary $\mathrm{T}$. sordida tended to increase but $\mathrm{T}$. cruzi infection remained low. These findings suggest that control of T. infestans using only insecticides (BHC and Deltamethrin) is difficult and expensive. Community participation is essential in programmes for the control of Chagas' disease. Consequently, the use of integrated measures is necessary in the control of Chagas' disease to encourage the active participation of afflicted communities, stimulated through educational programs.

Key words: Trypanosoma cruzi. Triatoma infestans. Triatoma sordida. Insecticides. Chagas' disease control.

\section{AGRADECIMENTOS}

À SUCAM - Divisão de Doença de Chagas, nas pessoas de: Antônio Carlos da Silveira, Tadayasu Sakamoto, C arlos Alberto Silveira Matos e, especialmente, aos abnegados guardas rurais, sem os quais nào teria sido possível a execuçào deste trabalho.

À Sra. Tânia Mara A. Campos, pela ajuda inestimável e presteza na análise estatistica.

\section{REFERÊNCIAS BIBLIOGRÁFICAS}

1. Dias E. Sugestões preliminares para um plano de erradicação dos transmissores domiciliares da doença de Chagas. Estado de São Paulo. Revista Médica Sul-Minas 2: 104-113, 1957 
Garcia-Zapata MT, Marsden PD, Virgens D, Penna R, Soares V, Brasil IA, Castro CN, Prata A, Macêdo V. O controle da transmissāo da doença de Chagas em Mambai-Goiás, Brasil (1982-1984). Revista da Sociedade Brasileira de Medicina Tropical 19: 219-225, Out-Dez, 1986.

2. Dias JCP. History and findings of the Bambui project. In: New Approaches in American Trypanosomiasis Research. Scientific Publication - PAHO 318: 338-339, 1975.

3. Forattini OP, Pereira OA, Rabello EX, Barata JMS, Santos JLS. Aspectos ecológicos da tripanosomiase americana. XVIII Desenvolvimento e ciclos anuais de colónias de $T$. infestans, $T$. sordida e $R$. neglectus em ecótopos artificiais no ambiente peri e intradomiciliar. Revista de Saúde Pública de São Paulo 17: 243-262, 1983.

4. Freitas JLP de, Ferreira OA, Duarte GG, Haddad D. Combate intensivo aos triatomíneos de hábitos domiciliares. Resultados obtidos em área restrita (Cassia dos Coqueiros, Municipio de Cajuru) do Estado de São Paulo, Brasil. Revista do Instituto de Medicina Tropical de Sāo Paulo 2: 100-107, 1960.

5. Garcia-Zapata MT. Vigilância epidemiológica no controle do Triatoma infestans em duas áreas do Estado de Goiás - Brasil. Tese de Mestrado. Universidade de Brasilia, Brasília - DF, Brasil, 1985.

6. Garcia-Zapata MT, Marsden PD, Virgens D, Prata AR, Macêdo V, Brasil IP, Matos CAS, Silveira AC. Sugestóes para a implantação de postos de informação e ataque triatomínico (PIAT), como uma nova alternativa nos programas de controle da doença de Chagas. Revista da Sociedade Brasileira de Medicina Tropical 17 (Sup.): 61, 1984.

7. Garcia-Zapata MT, Marsden PD, Virgens D, Prata AR, Macêdo V, Castro CN, Rocha M, Matos CAS, Silveira AC, Brasil IP. Avaliação de um programa educativo dentro de uma campanha de controle da doença de Chagas em Mambai - GO. Fase preliminar. Revista da Sociedade Brasileira de Medicina Tropical 17 (Sup.): $62,1984$.

8. Marsden PD. The control of Chagas' disease in Mambai - Brasil. The initial phases. Infection Control 2: 466470, 1981.

9. Marsden PD, Alvarenga NJ, Cuba CC, Shelley AJ, Costa $\mathrm{CH}$, Boreham PFL. Studies of the domestic ecology of Triatoma infestans by means of house demolition. Revista do Instituto de Medicina Tropical de São Paulo 21: 13-25, 1975.

10. Marsden PD, Penna RA. "Vigilance unit" for households subject to triatomine control. Transactions of the Royal Society of Tropical Medicine and Hygiene 76 : 790-792, 1982.

11. Marsden PD, Virgens D, Magalhães I, Tavares-Neto J, Ferreira R, Costa CNH, Macêdo V, Prata AR. Ecologia doméstica do Triatoma infestans em Mambai, Goiás Brasil. Revista do Instituto de Medicina Tropical de São Paulo 24: 364-375, 1982.

12. Marsden PD, Virgens D, Castro CN, Brasil IP, Ferreira R, Silveira AC, Matos CAS, Macêdo V, Prata A. The control of Chagas' disease transmission in Mambai, Goiás - Brasil (1980-1981). Revista da Sociedade Brasileira de Medicina Tropical 16: 189-195, 1983.

13. Organização Panamericana Sanitária. Situación de la enfermedad de Chagas en la region de las Américas. Boletim Epidemiológico OPAS 5: 5-9, 1984.

14. Piesman J, Sherlock IA. Sensibilidade de caixas de Gómez-Nuñez para a deteç̧ão de infestação doméstica com Panstrongylus megistus. Revista da Sociedade Brasileira de Medicina Tropical 17: 21-25, 1984.

15. Rocha e Silva EO, Guarita AF, Ishihata GK. Doença de Chagas: atividades de controle dos transmissores no Estado de São Paulo. Revista Brasileira de Malariologia e Doenças Tropicais 31: 99-119, 1979.

16. Schofield CJ, Mathews JNS. Theoretical approach to active dispersal and colonization of houses by Triatoma infestans. Journal of Tropical Medicine and Hygiéne, in press.

17. Sherlock IA, Muniz TM. Observaçōes sobre o combate ao $P$. megistus com BHC em área infestada do Estado da Bahia, Brasil. Revista Brasileira de Malariologia e Doenças Tropicais 26/27: 102, 1974/1975.

18. Sherlock IA, Piesman J. Controle da transmissão da doença de Chagas, pelo combate aos vetores, com bendiocarb, feito pelos habitantes. Revista da Sociedade Brasileira de Medicina Tropical 17: 67-72, 1984.

19. Soler CA, Schenone H, Reyes H. Problemas derivados de la reaparición de Triatoma infestans en viviendas desinsectadas y el concepto de reinfestación. Boletin Chileno de Parasitologia 24: 83-84, 1969.

20. Superintendência de Campanhas de Saúde Pública (SUC AM). Manual do Guarda de Inseticida. Centro de Documentação do Ministério da Saúde, Brasília - DF, 1975.

21. Superintendência de Campanhas de Saúde Pública. Divisão de Doença de Chagas. Manual de Normas Técnicas da Campanha de Controle da Doença de Chagas. Centro de Documentação do Ministério da Saúde, Brasília - DF, 1980.

22. World Health Organization. Epidemiological indicators. Meeting on epidemiological longitudisal studies on Chagas' disease. Rio de Janeiro, Brasil. Unpublished series WHO. TDR/EPICHALES/83. 3, 1983. 